\title{
Renormalization Mass Scale and Scheme Dependence in the Perturbative Contribution to Inclusive Semileptonic $b$ Decays
}

\author{
F.A. Chishtie ${ }^{1}$, D.G.C. McKeon ${ }^{2,3}$, and T.N. Sherry ${ }^{4}$ \\ ${ }^{1}$ Department of Applied Mathematics, The University of Western Ontario, \\ London, ON N6A 5B7, Canada \\ ${ }^{2}$ Department of Mathematics and Computer Science, Algoma University, \\ Sault Ste. Marie, ON P6A 2G4, Canada \\ ${ }^{3}$ School of Mathematics, Statistics and Applied Mathematics, National University \\ of Ireland Galway, University Road, Galway, Ireland H91 TK33
}

January 20, 2021

PACS No.: 11.10Hi

Key Words: renormalization scheme, b-quark decays email: fachisht@uwo.ca,dgmckeo2@uwo.ca, tom.sherry@nuigalway.ie

\begin{abstract}
We examine the perturbative calculation of the inclusive semi-leptonic decay rate $\Gamma$ for the $b$-quark, using mass-independent renormalization. To finite order of perturbation theory the series for $\Gamma$ will depend on the unphysical renormalization scale parameter $\mu$ and on the particular choice of mass-independent renormalization scheme; these dependencies will only be removed after summing the series to all orders. In this paper we show that all explicit $\mu$ dependence of $\Gamma$, through powers of $\ln (\mu)$, can be summed by using the renormalization group equation. We then find that this explicit $\mu$-dependence can be combined together with the implicit $\mu$-dependence of $\Gamma$ (through powers of both the running coupling $a(\mu)$ and the running $b$-quark mass $m(\mu))$ to yield a $\mu$-independent perturbative expansion for $\Gamma$ in terms of $a(\mu)$ and $m(\mu)$ both evaluated at a renormalization scheme independent mass scale $M$ which is fixed in terms of either the " $\overline{M S}$ mass" $\bar{m}_{b}$ of the $b$ quark or its pole mass $m_{\text {pole }}$. At finite order the resulting perturbative expansion retains a degree of arbitrariness associated with the particular choice of mass-independent renormalization scheme. We use the coefficients $c_{i}$ and $g_{i}$ of the perturbative expansions of the renormalization group functions $\beta(a)$ and $\gamma(a)$, associated with $a(\mu)$ and $m(\mu)$ respectively, to characterize the remaining renormalization scheme arbitrariness
\end{abstract}


of $\Gamma$. We further show that all terms in the expansion of $\Gamma$ can be written in terms of the $c_{i}$ and $g_{i}$ coefficients and a set of renormalization scheme independent parameters $\tau_{i}$. A second set of renormalization scheme independent parameters $\sigma_{i}$ is shown to play a very similar role in the perturbative expansion of $m_{\text {pole }}$ in terms of $m(\mu)$ and $a(\mu)$. We illustrate our approach by a perturbative computation of $\Gamma$ using the $\overline{M S}$ renormalization scheme. Two other particular mass independent renormalization schemes are briefly considered.

\section{Introduction}

Mass independent renormalization schemes $[1,2]$ are relatively easy to implement, but are often viewed as being "unphysical". This is particularly true when the process being considered involves a heavy particle such as a $b$ quark. In addition to this problem, perturbative results obtained using a mass independent renormalization scheme $(\mathrm{RS})$ depend on a non-physical scale parameter $\mu$. Furthermore, results at a finite order of perturbation theory can be altered by making a finite renormalization of the quantities that characterize the theory (masses, couplings and field strengths).

An analysis of the ambiguities associated with $\mu$ and the ambiguities in RS appear in ref. [8]. In this reference, it was proposed that the ambiguities could be resolved at finite order by applying the "Principle of Minimal Sensitivity". In ref. [3] the renormalization group (RG) equation is used to sum logarithmic corrections to various processes. One can use the RG functions at $n$-loop order to sum the $N^{n-1} L L$ corrections (leading-log, next-to-leading-log, etc.). Alternatively, one can sum all logarithmic corrections in terms of the log-independent corrections and the RG functions. This latter approach was used in refs. [4,5] to examine $R_{e^{+} e^{-}}$, the cross section for $e^{+} e^{-} \rightarrow$ hadrons and further processes were studied in [6]. It was found that upon summing all logarithmic contributions to $R_{e^{+} e^{-}}$the explicit dependence of $R_{e^{+} e^{-}}$on $\mu$ cancelled with its implicit dependence on $\mu$, leaving $R_{e^{+} e^{-}}$dependent only on the ratio $Q / \Lambda$ where $Q$ is the centre of mass energy and $\Lambda$ is a mass scale associated with the boundary value of the running coupling $a(\ln \mu / \Lambda)$. A set of RS invariant parameters $\tau_{i}$ was found, and $R_{e^{+} e^{-}}$was seen to be expressible in terms of $a(\ln Q / \Lambda)$ and $\tau_{i}$. The parameters $\tau_{i}$ are related to the RS invariant parameters found in refs. [8,25].

In this paper we examine the decay rate $\Gamma$ relevant for both the processes $b \rightarrow u \ell^{-} \bar{\nu}_{\ell}$ and $b \rightarrow c \ell^{-} \bar{\nu}_{\ell}$. These decays have both perturbative and non-perturbative contributions, and in this work we will restrict our attention to the ambiguities inherent in perturbative calculations involving $b$ quark decay. Addressing such an issue involves a massive parameter (the mass of the $b$ quark) contributing explicitly to $\Gamma$ which leads to a complication in the analysis as when using a "mass independent" RS, the mass now "runs"; this is different from the computation of $R_{e^{+} e^{-}}$where there is a fixed massive scale $Q$, the centre of mass energy, entering the calculation. (A calculation of $\Gamma$ which uses the pole mass [16] of the $b$ quark [3,7] rather than its running mass $m(\mu)$ is possible, but such a calculation does not use a mass independent RS.) 
It is possible to use the RG approach to carry out various partial summations of the perturbative expansion of the decay rate $\Gamma$. The first of these involves use of the RG equation to sum the $L L, N L L, \ldots N^{p} L L$ contributions to $\Gamma$ in terms of the $1,2, \ldots,(p+1)$ loop contributions to $\beta$ and $\gamma$, the RG functions associated with $a(\mu)$ and $m(\mu)$ respectively. A second approach is to sum all logarithmic contributions to $\Gamma$ so that $\Gamma$ is expressed in terms of its log independent part. One appealing feature of this log-summed form is that the explicit and implicit dependence on $\mu$ is again shown to cancel, much as it did in the analysis of $R_{e^{+} e^{-}}$. This removes one source of ambiguity in any subsequent calculation of the decay rate $\Gamma$.

Even within the mass independent renormalization schemes, there is a degree of RS ambiguity beyond the ambiguities arising from $\mu$. The renormalization scheme can be parameterized by the appropriate coefficients $c_{i}$ and $g_{i}$ in the loop expansion of the RG functions associated with the coupling constant and the anomalous mass dimension respectively [8,9]. The requirement that $\Gamma$ be RS independent leads to a set of RS invariant parameters $\tau_{i}$. Different RS's are considered, each leaving $\Gamma$ expressed in terms of these $\tau_{i}$, a mass parameter $M$ associated with the $b$ quark, and a QCD scale parameter $\Lambda$ as well as the parameters $c_{i}$ and $g_{i}$ appropriate for these $R S$ 's. In one of these schemes, the perturbative expansion for $\Gamma$ terminates after a finite number of terms, while in the other ('t Hooft-like scheme [10]) the perturbative expansions for the RG functions $\beta$ and $\gamma$ terminate.

We also apply RG summation to relate the pole mass [16] for the $b$ quark $m_{\text {pole }}$ to its running mass $m(\mu)$. We use this relation to show how $\Gamma$ can be found in terms of the physical, RS invariant mass scale, $m_{\text {pole }}$ in place of the mass parameter $M$.

\section{Renormalization Group Summation}

A perturbative evaluation of the amplitude $\Gamma$ for the semi-leptonic decay process $b \rightarrow u \ell^{-} \bar{\nu}_{\ell}$ or $b \rightarrow c \ell^{-} \bar{\nu}_{\ell}$ leads to the expression ${ }^{1}$

$$
\Gamma_{q}=\frac{G_{F}^{2}\left|V_{q b}\right|^{2}}{192 \pi^{3}}[m(\mu)]^{5} \sum_{n=0}^{\infty} \sum_{k=0}^{n} T_{n, k} a^{n}(\mu) \ln ^{k}\left(\frac{\mu}{m(\mu)}\right)
$$

where $m(\mu)$ is the running mass for the $b$ quark, $a(\mu)\left(=\alpha_{s}(\mu) / \pi\right)$ is the strong coupling, $q=c$ or $u$ as appropriate, and we assume five active flavours. We will work with the reduced decay width

$$
\Gamma=\Gamma_{q}\left(\frac{G_{F}^{2}\left|V_{q b}\right|^{2}}{192 \pi^{3}}\right)^{-1}
$$

\footnotetext{
${ }^{1}$ The expression for $\Gamma$ in eq. (1) is distinct from the way the solution to the RG equation of eq. (3) is usually presented [11] as it does not involve simply replacing the mass and coupling in a perturbative expression for $\Gamma$ by the running coupling and mass. This is discussed in more detail in ref. [12]. Eq. (1) is an ansatz for the solution to the RG equation that is motivated by knowing the form of results obtained from evaluating the $n$-loop Feynman diagrams needed to obtain the coefficients $T_{n}(k=0,1 . . n)$.
} 
throughout this paper. As the exact expression for $\Gamma$ is independent of the unphysical renormalization scale parameter $\mu$ we have the $\mathrm{RG}$ equation

$$
\mu \frac{d \Gamma}{d \mu}=0=\left(\mu \frac{\partial}{\partial \mu}+\beta(a) \frac{\partial}{\partial a}+m \gamma(a) \frac{\partial}{\partial m}\right) \Gamma
$$

where

$$
\beta(a)=\mu \frac{\partial a}{\partial \mu}=-b a^{2}\left(1+c a+c_{2} a^{2}+\ldots\right)
$$

and

$$
m \gamma(a)=\mu \frac{\partial m}{\partial \mu}=m f a\left(1+g_{1} a+g_{2} a^{2}+\ldots\right) .
$$

Eq. (1) ensures that the explicit dependence on $\Gamma$ on $\mu$ through $\ln (\mu)$ and its implicit dependence through $a(\mu)$ and $m(\mu)$ cancels. Approximating the computation of $\Gamma$ up to, and including, $N$-loop Feynman diagrams involves truncating the summation process in eq. (1)

$$
\Gamma^{(N)}(\mu)=[m(\mu)]^{5} \sum_{n=0}^{N} \sum_{k=0}^{n} T_{n, k} a^{n}(\mu) \ln ^{k}\left(\frac{\mu}{m(\mu)}\right) .
$$

This $N$-loop approximation is highly dependent on the arbitrary renormalization scale $\mu$, both explicitly and implicitly, as illustrated in ref. [3] for $N=3$. No convincing argument for specifying a particular value of $\mu$ in $\Gamma^{(N)}(\mu)$ has gained acceptance.

In ref. [3] we also considered a re-ordering of the summation in eq. (1) so that eq. (3) could be used to sum the contributions to $\Gamma$ of all leading-logarithms $(L L)$, next-to-leading-logarithms ( $N L L)$ etc., which led to a lessening of the $\mu$-dependence in a finite order approximation; however, this approach did not totally eliminate dependence on $\mu$. The $N^{P} L L$ sum contributing to $\Gamma$ receives contributions from all $T_{p+k, k}(k \geq 0)$ with $T_{p, 0}$ providing a boundary value, and from the $\beta$ and $\gamma$ functions up to order $p+1$ in the loop expansion.

In this paper we use a different way of organizing the sums in eq. (1). In this approach, we will sum all log dependent contributions to $\Gamma$. The $R G$ equation will make it possible to express all these $\log$ dependent contributions in terms of log independent parts in such a way that all dependence on $\mu$ is completely removed. This approach is used in the discussion of $R_{e^{+} e^{-}}$in refs. [4,5]. However, the summation is more complicated here than it was for $R_{e^{+}} e^{-}$. This is because eq. (1) shows that $\Gamma$ does not directly depend on a physical mass parameter which would be analogous to the centre of mass energy $Q$ that occurs in refs. [4,5]. Instead a "running mass" $m(\mu)$ associated with the renormalized mass of the $b$ quark appears. For $R_{e^{+} e^{-}}$one can very simply sum all of the log-dependent contributions to show immediately that all $\mu$ dependence is removed. This result is also possible for $\Gamma$, but it proves to be more awkward to sum all of its log-dependent pieces.

We begin by defining

$$
A_{n}(a(\mu))=\sum_{k=0}^{\infty} T_{n+k, n} a(\mu)^{n+k}
$$


so that eq. (1) leads to

$$
\Gamma=m^{5}(\mu) \sum_{n=0}^{\infty} A_{n}(a(\mu)) \ell^{n}
$$

where now $\ell=\ln (\mu / m(\mu))$. We recognize $A_{n}(a(\mu))$ in eq. (7) as the sum of all the coefficients of $\ell^{n}$ in the perturbative expansion of $\Gamma$ in eq. (1). In particular, $A_{0}(a(\mu))$ contains all of the $\log$ independent parts of $\Gamma$. If eq. (8) is substituted into eq. (3) we find

$$
A_{n}(a(\mu))=\frac{-1}{n}\left[\hat{\beta}(a(\mu)) \frac{\partial}{\partial a}+5 \hat{\gamma}(a(\mu))\right] A_{n-1}(a(\mu))
$$

where

$$
\begin{aligned}
& \hat{\beta}=\beta /(1-\gamma), \\
& \hat{\gamma}=\gamma /(1-\gamma) .
\end{aligned}
$$

We now define

$$
E(a(\mu))=\exp \left[\int_{0}^{a(\mu)} d x \frac{\gamma(x)}{\beta(x)}+\int_{0}^{K} d x \frac{f x}{b x^{2}(1+c x)}\right]
$$

where $K$ is some cut off. The second integral in eq. (11) is a RS independent infinite constant whose role is to ensure that the argument of the exponential is finite.

We also note that solutions to eqs. $(4,5)$ can be written as

$$
\ln \left(\frac{\mu}{\Lambda}\right)=\int_{0}^{a(\mu)} \frac{d x}{\beta(x)}+\int_{0}^{K} \frac{d x}{b x^{2}(1+c x)}
$$

and

$$
m(\mu)=\mathbb{M E}(a(\mu))
$$

where $\Lambda$ and $M$ are scale dependent quantities used to define boundary conditions on eqs. $(4,5)$. We note that in eqs. $(12,13)$ a change in $K$ can be absorbed into changes in $\Lambda$ and $\mathbb{M}$. The scale parameter $M$ is closely related to a scalar parameter that is RS invariant that was previously used in ref. [26]. In refs. [8,9], $K$ is taken to be infinite. (Below we will often use $a(\mu)$ to denote $a\left(\ln \frac{\mu}{\Lambda}\right)$. Similarly, the $\mu$ dependence of any dimensionless quantity, such as $E$, will be written $E(a(\mu))$ but will be understood to mean $E(a(\ln \mu / \Lambda))$. )

We now can re-express eq. (9) in the form

$$
B_{n}(a(\mu))=\frac{-1}{n} \hat{\beta}(a(\mu)) \frac{\partial}{\partial a} B_{n-1}(a(\mu))
$$

where

$$
B_{n}(a(\mu))=E^{5}(a(\mu)) A_{n}(a(\mu)) .
$$


We now define an auxiliary quantity $\eta$ so that

$$
\frac{\partial}{\partial \eta}=\hat{\beta}(a) \frac{\partial}{\partial a}
$$

so that by eq. (10a)

$$
\eta(a(\mu))=\int_{0}^{a(\mu)} d x \frac{1-\gamma(x)}{\beta(x)}+\int_{0}^{K} d x \frac{1-f x}{b x^{2}(1+c x)} .
$$

Together eqs. $(14,16)$ show that

$$
B_{n}(a(\mu))=\frac{-1}{n} \frac{\partial}{\partial \eta} B_{n-1}(a(\mu))
$$

or upon iteration,

$$
=\frac{(-1)^{n}}{n !} \frac{\partial^{n}}{\partial \eta^{n}} B_{0}(a(\mu)) .
$$

Eqs. $(8,15,19)$ together lead to

$$
\Gamma=m^{5}(\mu) E^{-5}(a(\mu)) \sum_{n=0}^{\infty} \frac{(-\ell)^{n}}{n !}\left(\frac{\partial}{\partial \eta}\right)^{n} B_{0}(a(\mu)) .
$$

Eq. (13) results in eq. (20) becoming

$$
\Gamma=M^{5} B_{0}(a(\eta-\ell)) .
$$

However, we now see by eqs. (11-13,17), that

$$
\eta-\ell=\ln \left(\frac{M}{\Lambda}\right)
$$

which is $\mu$ independent. We now can combine eqs. $(15,21,22)$ to obtain

$$
\begin{array}{r}
\Gamma=M^{5} E^{5}\left(\ln \frac{M}{\Lambda}\right) A_{0}\left(a\left(\ln \frac{M}{\Lambda}\right)\right) \\
=m^{5}\left(\ln \frac{M}{\Lambda}\right) A_{0}\left(a\left(\ln \frac{M}{\Lambda}\right)\right)
\end{array}
$$

We see that $\Gamma$ is now expressed in terms of its log-independent contribution $A_{0}$ and that it is $\mu$ independent. Even when we are restricted to a finite number of terms in the expansion of $A_{0}\left(a\left(\ln \frac{M}{\Lambda}\right)\right)$

$$
\sum_{k=0}^{N} T_{k, 0} a\left(\ln \frac{M}{\Lambda}\right)^{k}
$$

the resulting approximation to $\Gamma$ is still $\mu$-independent. However it retains a residual RS dependence as will be discussed in the following section. It is interesting that eq. (23) can be obtained from eq. 
(1) by dropping all terms involving $\left(\ln \frac{\mu}{\Lambda}\right)^{k}(k \geq 1)$ and then setting $\mu=M$. The exact expression for the mass scale $\mathbb{M}$ appearing throughout eq. (23) will be shown to be a RS independent (though unphysical) quantity. However, in section four it will be shown that $M$ can be expressed in terms of the pole mass of the $b$ quark, $m_{\text {pole }}$, a physical quantity that is RS invariant.

In eq. (23b), $A_{0}\left(a\left(\ln \frac{M}{\Lambda}\right)\right)$ is to be evaluated by computing Feynman diagrams and is expressed as a power series in $a\left(\ln \frac{M}{\Lambda}\right)$. The convergence of such series is not clearly understood; it is generally accepted that they are asymptotic series and that divergences due to "renormalons" [10,13] can arise. The problem of convergence of the perturbative series in $a$ is not a focus of our work; our focus is on the ambiguities that arise to a finite order in a perturbative expansion when using mass independent renormalization. We have shown that the ambiguity residing in eq. (1) due to the presence of $\mu$ when this series is truncated can be fixed by using the RG equation to sum those logarithms containing explicit dependence on $\mu$. Other approaches to eliminating ambiguities arising in the computation of perturbative effects are "The Principle of Maximal Conformality" (PMC) [27,28] and "Complete Renormalization Group Improvement" (CORGI) [29,31]. A comparison of CORGI with the approach used here appears in [30]. We now consider ambiguities residing in the choice of RS.

\section{Renormalization Scheme Dependence}

It has been established that the RS ambiguities within mass independent RS can be parameterized by the mass scale $\mu$ as well as the coefficients $c_{i}(i \geq 2), g_{i}(i \geq 1)$ in eqs. $(4,5)$. In a recent paper [14], we examined how the running coupling $a(\mu)$ and the running mass $m(\mu)$ vary with RS.

Focussing first on $\mu$, the scale dependence, we found

$$
\begin{aligned}
a(\mu) & =a\left(\mu^{\prime}\right)\left[1+b \lambda a\left(\mu^{\prime}\right)+b \lambda(c+b \lambda) a^{2}\left(\mu^{\prime}\right)+b \lambda\left(c_{2}+\frac{5}{2} b c \lambda+b^{2} \lambda^{2}\right) a^{3}\left(\mu^{\prime}\right)+\ldots\right] \\
m(\mu) & =m\left(\mu^{\prime}\right)\left[1-f \lambda a\left(\mu^{\prime}\right)-f \lambda\left(g_{1}-\frac{1}{2}(f-b) \lambda\right) a^{2}\left(\mu^{\prime}\right)\right. \\
& \left.-f \lambda\left(g_{2}+\left(b g_{1}+\frac{b c}{2}-f g_{1}\right) \lambda+\frac{1}{3}(f-b)\left(\frac{f}{2}-b\right) \lambda^{2}\right) a^{3}\left(\mu^{\prime}\right)+\ldots\right]
\end{aligned}
$$

where $\lambda=\ln \left(\mu^{\prime} / \mu\right)$. These results tell us how the running coupling and running mass at two different scales are related, all within one RS. 
Turning now to the dependence on the $c_{i}$ and $g_{i}$ parameters, it was shown in refs. $[8,9]$ that

$$
\begin{aligned}
\frac{\partial a}{\partial c_{i}} & =B_{i}(a) \\
& =-b \beta(a) \int_{0}^{a} d x \frac{x^{i+2}}{\beta^{2}(x)} \\
& =a^{i+1}\left(W_{0}^{i}+W_{1}^{i} a+W_{2}^{i} a^{2}+\ldots\right) \\
\frac{1}{m} \frac{\partial m}{\partial c_{i}} & =\Gamma_{i}^{c}(a) \\
& =\frac{\gamma(a)}{\beta(a)} B_{i}(a)+b \int_{0}^{a} d x \frac{x^{i+2} \gamma(x)}{\beta^{2}(x)} \\
& =a^{i}\left(U_{0}^{i}+U_{1}^{i} a+U_{2}^{i} a^{2}+\ldots\right) \\
\frac{1}{m} \frac{\partial m}{\partial g_{i}} & =\Gamma_{i}^{g}(a) \\
& =f \int_{0}^{a} d x \frac{x^{i+1}}{\beta(x)} \\
& =a^{i}\left(V_{0}^{i}+V_{1}^{i} a+V_{2}^{i} a^{2}+\ldots\right)
\end{aligned}
$$

and

$$
\frac{\partial a}{\partial g_{i}}=0
$$

The first few $W_{j}^{i}, U_{j}^{i}$ and $V_{j}^{i}$ coefficients can be found in ref. [14]. We have also derived the relationship between the running couplings $a\left(\mu, c_{i}\right)$ and the running masses $m\left(\mu, c_{i}, g_{i}\right)$ at the same renormalization scale but at different values of the parameters $c_{i}$ and $g_{i}$. If

$$
\begin{aligned}
a^{\prime} & =a\left(\mu, c_{i}^{\prime}\right), a=a\left(\mu, c_{i}\right) \\
m^{\prime} & =m\left(\mu, c_{i}^{\prime}, g_{i}^{\prime}\right), m=m\left(\mu, c_{i}, g_{i}\right)
\end{aligned}
$$

then the first few terms in these relationships are $\left(\rho \equiv \frac{f}{b}\right)$

$$
\begin{aligned}
& a^{\prime}=a\left[1+\left(c_{2}^{\prime}-c_{2}\right) a^{2}+\frac{1}{2}\left(c_{3}^{\prime}-c_{3}\right) a^{3}+\ldots\right] \\
& m^{\prime}=m\left[1+\rho\left(g_{1}-g_{1}^{\prime}\right) a+\frac{\rho}{2}\left(g_{2}-g_{2}^{\prime}+c_{2}-c_{2}^{\prime}\right.\right. \\
& \left.\left.-c\left(g_{1}-g_{1}^{\prime}\right)+\rho\left(g_{1}-g_{1}^{\prime}\right)^{2}\right) a^{2}+\ldots\right]
\end{aligned}
$$

Further terms in these relationships can be found in ref. [14].

In ref. [14] we also reorganized the summations in eq. (25) to end up with

$$
\begin{aligned}
a(\mu) & =\sum_{n=0}^{\infty} X_{n}\left(a\left(\mu^{\prime}\right) \lambda\right) a\left(\mu^{\prime}\right)^{n+1} \\
m(\mu) & =m\left(\mu^{\prime}\right) \sum_{n=0}^{\infty} Y_{n}\left(a\left(\mu^{\prime}\right) \lambda\right) a\left(\mu^{\prime}\right)^{n}
\end{aligned}
$$


where, as before, $\lambda=\ln \left(\mu^{\prime} / \mu\right)$. $X_{n}$ and $Y_{n}$ have been computed iteratively up to $n=4$ corresponding to the sums of all leading-logarithms, next-to-leading-logarithms ... (next-to) ${ }^{4}$-leading-logarithms. In particular, the first three solutions are

$$
\begin{aligned}
& X_{0}(\xi)=\frac{1}{w} \quad(w \equiv 1-b \xi) \\
& X_{1}(\xi)=\frac{-c \ln w}{w^{2}} \\
& X_{2}(\xi)=\frac{c^{2}\left(\ln ^{2} w-\ln w+w-1\right)-c_{2}(w-1)}{w^{3}}
\end{aligned}
$$

$$
\begin{aligned}
Y_{0}(\xi) & =w^{\rho} \\
Y_{1}(\xi) & =\rho w^{\rho-1} \quad\left[c(\ln w)+(1-w)\left(c-g_{1}\right)\right] \\
Y_{2}(\xi) & =\frac{\rho}{2} w^{-2+\rho}\left\{-c^{2}(1-\rho) \ln ^{2} w+\left[-2 \rho\left(c-g_{1}\right)(w-1)+2 g_{1}\right] c \ln w\right. \\
& \left.+(w-1)\left[\left(c^{2}-c_{2}+\rho\left(c-g_{1}\right)^{2}\right)(w-1)+\left(g_{2}-c g_{1}\right)(w+1)\right]\right\} .
\end{aligned}
$$

We note that $X_{n}$ depends on $c_{m}\left(c_{0} \equiv b, c_{1} \equiv c\right)$ for $m=0,1 \ldots n$ and $Y_{n}$ depends on $c_{m}$ and $g_{m}\left(g_{0} \equiv f\right)$ for $m=0,1 \ldots n$. In general, $c_{m}$ and $g_{m}$ are computed through evaluation of Green's functions with $1,2 \ldots(m+1)$ loops.

The mass parameter $M$ was introduced in eq. (13). Written in the form

$$
M=\frac{m(\mu)}{E(a(\mu))}
$$

it appears to be a RS dependent quantity. However, by direct differentiation with respect to each of the RS parameters $c_{i}$ and $g_{i}$, we show that the exact expression for $M$ is, in fact, RS independent.

$$
\begin{aligned}
\frac{\partial M}{\partial c_{i}} & =m(\mu)\left[\Gamma_{i}^{c}(a)-B_{i}(a) \frac{\gamma(a)}{\beta(a)}-\int_{0}^{a} d x \frac{b x^{i+2} \gamma(x)}{\beta^{2}(x)}\right] E^{-1}(a(\mu))=0 \\
\frac{\partial M}{\partial g_{i}} & =m(\mu)\left[\Gamma_{i}^{g}(a)-\int_{0}^{a} d x \frac{f x^{i+1}}{\beta(x)}\right] E^{-1}(a(\mu))=0 .
\end{aligned}
$$

By a similar computation, it also follows that

$$
\mu \frac{\partial M}{\partial \mu}=0 .
$$

It should be noted however that even though an exact expression for $M$ given by eq. (31) is independent of $\mu, c_{i}$ and $g_{i}$, any approximation for $M$ based on a finite number of terms contributing to $m(\mu)$ or $E(a(\mu))$ will have some dependence on these parameters.

The full resummed expression of eq. (23) for the decay rate $\Gamma$ is $\mathrm{RS}$ independent. However, $a\left(\ln \frac{M}{\Lambda}\right)$ and $m\left(\ln \frac{\mathbb{M}}{\Lambda}\right)$ both display RS dependence through the $c_{i}$ and $g_{i}$ according to eq. (27). 
It follows that the expansion coefficients $T_{n} \equiv T_{n, 0}$ must also be explicitly RS dependent. This RS dependence can be deduced from

$$
\begin{aligned}
& \frac{\partial \Gamma}{\partial c_{i}}=0=m^{5} \sum_{n=0}^{\infty}\left[5 \Gamma_{i}^{c}(a) T_{n} a^{n}+\frac{\partial T_{n}}{\partial c_{i}} a^{n}+n B_{i}(a) T_{n} a^{n-1}\right] \quad(i \geq 2) \\
& \frac{\partial \Gamma}{\partial g_{i}}=0=m^{5} \sum_{n=0}^{\infty}\left[5 \Gamma_{i}^{g}(a) T_{n} a^{n}+\frac{\partial T_{n}}{\partial g_{i}} a^{n}\right] \quad(i \geq 1)
\end{aligned}
$$

where $a \equiv a\left(\ln \frac{M}{\Lambda}\right)$ and $m \equiv m\left(\ln \frac{M}{\Lambda}\right)$, by using the explicit form of the $W_{n}^{i}, U_{n}^{i}$ and $V_{n}^{i}$ expansion coefficients referred to earlier. In particular, we have $U_{0}^{2}=-\rho / 2, V_{0}^{2}=-\rho / 2, V_{0}^{1}=-\rho$ and $V_{1}^{1}=c \rho / 2$. For $n=0,1,2$ we find that

$$
\begin{array}{ll} 
& \frac{\partial T_{0}}{\partial c_{i}}=0 \\
& \frac{\partial T_{1}}{\partial c_{i}}=0 \\
& \frac{\partial T_{2}}{\partial c_{i}}+5 \delta_{2}^{i}\left(T_{0} U_{0}^{i}\right)=0 \\
\frac{\partial T_{0}}{\partial g_{i}}=0 & \frac{\partial T_{1}}{\partial g_{i}}+5 \delta_{1}^{i}\left(T_{0} V_{0}^{i}\right)=0 \\
\frac{\partial T_{2}}{\partial g_{i}}+5 \delta_{2}^{i}\left(T_{0} V_{0}^{i}\right)+5 \delta_{1}^{i}\left(V_{0}^{1} T_{1}+V_{1}^{1} T_{0}\right)=0 .
\end{array}
$$

Solving these equations leads to

$$
\begin{aligned}
& T_{0}=\tau_{0} \\
& T_{1}=\tau_{1}+5 \rho \tau_{0} g_{1} \\
& T_{2}=\tau_{2}+\frac{5 \rho}{2}\left(\tau_{0} c_{2}+\tau_{0} g_{2}-c \tau_{0} g_{1}+2 \tau_{1} g_{1}+5 \rho \tau_{0} g_{1}^{2}\right)
\end{aligned}
$$

where $\tau_{0}, \tau_{1}, \tau_{2}$ are constants of integration and, consequently, RS independent. An iterative analysis of eq. (33) will generate the explicit solutions for $T_{n}(n \geq 3)$ in terms of the $c_{i}$ and $g_{i}(i=0,1 \ldots n)$ expansion coefficients and further RS independent variables $\tau_{n}(n \geq 3)$. The $\tau_{i}$ are related to the RS invariants derived in ref. [25].

Since $\tau_{0}, \tau_{1} \ldots$ are RS independent, their values can be identified in any RS from a perturbative calculation of the decay rate $\Gamma$ in eq. (23). If an explicit evaluation of $T_{n}, c_{i}$ and $g_{i}$ has been carried out to $\mathrm{N}^{\text {th }}$ order in perturbation theory through the evaluation of Feynman diagrams using some mass-independent RS, such as $\overline{M S}[2,15]$, then $\tau_{0}, \tau_{1} \ldots \tau_{N}$ can be deduced for this process by 
availing eqs. (36) above and their higher order iterative companion equations. It is interesting that the decay rate expansion coefficient $T_{n}$, in general, is computed by a calculation involving $n$-loop Green's functions. However, the identification of the value of $\tau_{n}$ involves $c_{i}, g_{i}(i=0,1 \ldots n)$ where $c_{n}$ and $g_{n}$ are obtained by considering $n+1$ loop Green's functions. Furthermore, identification of the decay rate expansion coefficient $T_{n}$, under a change of RS, involves alterations dependent on the same set of parameters, of which $c_{n}$ and $g_{n}$ are again obtained by considering $(n+1)$-loop Green's functions.

\section{The Pole Mass and the Running Mass}

In eq. (23) we have an expression for $\Gamma$ that depends on a mass scale $M$ which is essentially a boundary value for the equation for the running mass $m(\mu)$ of eq. (5). We will now relate this mass scale $M$ to the pole mass $m_{\text {pole }}$ of the $b$ quark. This pole mass is a RS independent, gauge invariant and infrared finite quantity [16]. Since quarks are always in a bound state, one cannot directly measure this pole mass; it is a quantity that is realized in perturbation theory. In a number of papers the self energy of the quark is discussed in detail and from this the relationship between $m_{\text {pole }}$ and $m(\mu)$ can be derived [17,35] (see also ref. [36]). From this, a relation can be derived between $M$ and $m_{\text {pole }}$.

If one uses a mass independent RS, the inverse of the renormalized quark propagator has the form

$$
S^{-1}\left(p^{\mu}, m(\mu)\right)=A\left(p^{2}, m(\mu)\right) \not b-m(\mu) B\left(p^{2}, m(\mu)\right) ;
$$

the pole mass is to be the location of the zero of this inverse propagator

$$
\lim _{\not x \rightarrow m_{\mathrm{pole}}} S^{-1}\left(p^{\mu}, m(\mu)\right)=0 .
$$

This results in an expansion

$$
m_{\text {pole }}=m(\mu) \sum_{n=0}^{\infty} \sum_{k=0}^{n} S_{n, k} a(\mu)^{n} L^{k}
$$

where $S_{0,0}=1$ and $L=\ln \left(\frac{\mu}{m_{\text {pole }}}\right)$. The coefficients $S_{n, k}$ in eq. (39) are also known numerically to four loop order [35]. In ref. [18] it is pointed out that for the $b$ quark, this expansion for the pole mass indicates that the relation between $m_{\text {pole }}$ and $m(\mu)$ is likely to be affected by divergences due to renormalons, beginning at third order, and that it might be more appropriate to use $m(\mu)$ rather than $m_{\text {pole }}$ when discussing the $b$ quark.

The structure of the equation relating $m_{\text {pole }}$ to $m(\mu)$ is very similar to that of eq. (1) for the decay width $\Gamma$. We can now reorganize the summations in eq. (39) in a similar manner to the analysis of section two. 
We define (as in eq. (7))

$$
F_{n}(a(\mu))=\sum_{k=0}^{\infty} S_{n+k, n} a(\mu)^{n+k}
$$

so that

$$
m_{\text {pole }}=m(\mu) \sum_{n=0}^{\infty} F_{n}(a(\mu)) L^{n} .
$$

We know that $m_{\text {pole }}$ is $\mu$ independent so that

$$
\mu \frac{d m_{\text {pole }}}{d \mu}=0=\left(\mu \frac{\partial}{\partial \mu}+\beta(a) \frac{\partial}{\partial a}+m \gamma(a) \frac{\partial}{\partial m}\right)\left[m \sum_{n=0}^{\infty} F_{n}(a) L^{n}\right] .
$$

This can be used to show that

$$
F_{n+1}(a)=-\frac{1}{n+1}\left(\beta(a) \frac{\partial}{\partial a}+\gamma(a)\right) F_{n}(a) .
$$

With $E$ defined in eq. (11) and

$$
\phi_{n}=E F_{n}
$$

then by eq. (43)

$$
\phi_{n+1}(a)=-\frac{1}{n+1} \beta(a) \frac{d}{d a} \phi_{n}(a)
$$

or

$$
\phi_{n+1}\left(a\left(\ln \frac{\mu}{\Lambda}\right)\right)=-\frac{1}{n+1} \frac{d}{d\left(\ln \frac{\mu}{\Lambda}\right)} \phi_{n}\left(a\left(\ln \frac{\mu}{\Lambda}\right)\right) .
$$

We thus find that

$$
\begin{gathered}
m_{\text {pole }}=m(\mu) E^{-1}(a(\mu)) \sum_{n=0}^{\infty} \frac{(-L)^{n}}{n !} \frac{d^{n}}{d \ln \left(\frac{\mu}{\Lambda}\right)^{n}} \phi_{0}\left(a\left(\ln \frac{\mu}{\Lambda}\right)\right) \\
=M \phi_{0}\left(a\left(\ln \frac{\mu}{\Lambda}-L\right)\right),
\end{gathered}
$$

or by eqs. (14) and (13)

$$
\begin{aligned}
m_{\text {pole }} & =M E\left(a\left(\ln \frac{m_{\text {pole }}}{\Lambda}\right)\right) F_{0}\left(a\left(\ln \frac{m_{\text {pole }}}{\Lambda}\right)\right) \\
& =m\left(\ln \frac{m_{\text {pole }}}{\Lambda}\right) F_{0}\left(a\left(\ln \frac{m_{\text {pole }}}{\Lambda}\right)\right) .
\end{aligned}
$$

The resummations carried out on the all-orders expansion of eq. (39) lead to eq. (49b) in which the b-quark pole mass is related to the running b-quark mass evaluated at the pole mass and the all orders series

$$
F_{0}\left(a\left(\ln \frac{m_{\text {pole }}}{\Lambda}\right)\right)=\sum_{n=0}^{\infty} S_{n, 0} a^{n}\left(\ln \frac{m_{\text {pole }}}{\Lambda}\right)
$$


As a by-product of this analysis, we find a second approach to the identification of the mass scale $M$ for use in the decay rate expansion of eq. (23b) and via eqs. (49a,b) to obtain

$$
M=\frac{m_{\text {pole }}}{E\left(a\left(\ln \frac{m_{\text {pole }}}{\Lambda}\right)\right) F_{0}\left(a\left(\ln \frac{m_{\text {pole }}}{\Lambda}\right)\right)}
$$

Availing eq. (51) for $M$ allows us to find the decay rate $\Gamma$ in terms of the pole mass and the running coupling evaluated at the pole mass.

It is now possible to examine the RS dependency of the expansion coefficients $S_{n} \equiv S_{n, 0}$ of the function $F_{0}$ defined in eq. (40). We find that since $m_{\text {pole }}$ in eq. (49) is RS invariant, then

$$
\mu \frac{d m_{\text {pole }}}{d \mu}=0=\frac{d m_{\text {pole }}}{d c_{i}}=\frac{d m_{\text {pole }}}{d g_{i}} .
$$

Using eqs. $(40,49)$ in eqs. $(52 b, c)$ leads to

$$
\begin{aligned}
& m \sum_{n=0}^{\infty}\left[\Gamma_{i}^{c}(a) S_{n} a^{n}+\frac{\partial S_{n}}{\partial c_{i}} a^{n}+n B_{i}(a) S_{n} a^{n-1}\right]=0, i \geq 2 \\
& m \sum_{n=0}^{\infty}\left[\Gamma_{i}^{g}(a) S_{n} a^{n}+\frac{\partial S_{n}}{\partial g_{i}} a^{n}\right]=0,(i \geq 1)
\end{aligned}
$$

where $a=a\left(\ln \frac{m_{\text {pole }}}{\Lambda}\right)$ and $m=m\left(\ln \frac{m_{\text {pole }}}{\Lambda}\right)$. For $n=0,1,2$ we find

$$
\frac{\partial S_{0}}{\partial c_{i}}=0, \quad \frac{\partial S_{1}}{\partial c_{i}}=0, \quad \frac{\partial S_{2}}{\partial c_{i}}-\frac{\rho}{2} S_{0} \delta_{2}^{i}=0
$$

and

$$
\frac{\partial S_{0}}{\partial g_{i}}=0, \quad \frac{\partial S_{1}}{\partial g_{i}}-\rho S_{0} \delta_{1}^{i}=0, \quad \frac{\partial S_{2}}{\partial g_{i}}+\left(-\rho S_{1}+\frac{\rho c}{2} S_{0}\right) \delta_{1}^{i}-\frac{\rho}{2} S_{0} \delta_{2}^{i}=0
$$

Solving these equations leads to

$$
\begin{gathered}
S_{0}=\sigma_{0} \\
S_{1}=\sigma_{1}+\rho \sigma_{0} g_{1}^{2} \\
S_{2}=\sigma_{2}+\frac{\rho}{2}\left(\sigma_{0} c_{2}+2 \sigma_{1} g_{1}-c \sigma_{0} g_{1}+2 \rho g_{2} g_{1} \sigma_{0}+\sigma_{0} g_{2}\right)
\end{gathered}
$$

In eq. (56), the quantities $\sigma_{i}$ are constants of integration and thus are RS invariants. Similar expressions can be found for all $S_{n}(n \geq 0)$. It is again interesting to note the relationship between expansion coefficients (in this case, those of the pole mass $S_{n}(n \geq 0)$ and RS invariant parameters $\left.\sigma_{n}(n>0)\right)$. The $S_{n}$ coefficients are found by computing n-loop Feynman integrals. However, the identification of the values of $\sigma_{n}(n>0)$ involves $c_{i}$ and $g_{i}(i=0,1, \ldots, n)$ where $c_{n}$ and $g_{n}$ are obtained by consideration of $(n+1)$-loop Feynman integrals. Furthermore, using eqs. (56a-c) and their iterative companion equations to determine the $n$-loop expansion coefficients $S_{n}$ for the pole 
mass (when relating it to the running mass and running coupling in a different RS), we see that the alterations of the $S_{n}$ involve changing the parameters $c_{n}$ and $g_{n}$ which are obtained from $(n+1)$-loop Feynman integrals. This dependence of the $n$-loop quantity $S_{n}$ on $(n+1)$-loop parameters $\left(c_{n}, g_{n}\right)$ is much like the dependence of $T_{n}$ on $\left(c_{n}, g_{n}\right)$ noted after eq. (36).

If it were possible to make an ansatz for $S_{n}$ on the parameters $c_{i}$ and $g_{i}$ that characterizes the RS, then summation such as the one that leads to the $\mu$-independent result of eq. (49b) would be feasible and all dependence on the RS parameters $c_{i}$ and $g_{i}$ would cancel. It is quite possible that the RS independent result would be identical to what would be obtained if we were to get $c_{i}=g_{i}=0$ at the outset so that $S_{n}=\sigma_{n}$, the RS independent parameters arising in eq. (56).

\section{Evaluation of $\Gamma$}

In order to illustrate how the formalism of the preceding sections can be applied, we now use it to compute $\Gamma$ in perturbation theory within the $\overline{M S}$ RS. Two other interesting RS will also be considered.

From ref. [7,18] we know that the coefficients $T_{n} \equiv T_{n, 0}$ appearing in eq. (1) are for $n=0,1,2$ (for $S U(3)$ with five flavours)

$$
\begin{gathered}
T_{0}=1 \\
T_{1}=\frac{65}{6}-\frac{2 \pi^{2}}{3} \\
T_{2}=\frac{3763495}{23328}-\frac{38 \pi^{2} \ln 2}{27}-\frac{35453 \pi^{2}}{2916}-\frac{3259 \zeta(3)}{81}+\frac{289 \pi^{4}}{648}
\end{gathered}
$$

when using $\overline{M S}$.

The known exact $\overline{M S}$ values up to two-loop are now used to find the first few terms in the perturbative expansion for $A_{0}\left(a\left(\ln \frac{M}{\Lambda}\right)\right)$

$$
A_{0} \approx T_{0} a+T_{1} a^{2}+T_{2} a^{3}
$$

appearing in eq. (23). (Via Padé approximation, $T_{3}$ can be estimated [19,20].) To find $\Gamma$ itself in eq. (23), we need first to determine $M$. By eq. (32) we know that the exact expression for $\mathbb{M}$ is independent of $\mu, c_{i}$ and $g_{i}$, so that it is RS independent, and consequently we estimate $M$ by using perturbative expansions for $m(\mu)$ and $E(a(\mu))$ in the $\overline{M S}$ RS. Since we are using perturbative expansions to find $M$, what we will use will retain dependence on $\mu$ and the RS used, even though the exact expression for $M$ is $\mu$ and $\mathrm{RS}$ independent. 
From eq. (11), as $K \rightarrow \infty$, the two loop estimate for $E$ is given by

$$
\begin{aligned}
E^{(2)}(a) & =\exp \left[\int_{0}^{a} d x \frac{f x\left(1+g_{1} x\right)}{-b x^{2}(1+c x)}+\int_{0}^{\infty} d x \frac{f x}{b x^{2}(1+c x)}\right] \\
& =\left[\frac{(1+c a)^{-1+g_{1} / c}}{c a}\right]^{f / b}
\end{aligned}
$$

while at three loop order, we also provide an analytic expression,

$$
E^{(3)}(a)=\exp \left[\int_{0}^{a} d x \frac{f\left(1+g_{1} x+g_{2} x^{2}\right)}{-b x^{2}\left(1+c x+c_{2} x^{2}\right)}+\int_{0}^{\infty} d x \frac{f x}{b x^{2}(1+c x)}\right]
$$

which for $4 c_{2}-c^{2}>0$ (which is satisfied in our situation) leads to

$$
\begin{aligned}
\ln E^{(3)}(a)= & -\frac{f}{b} \ln (c a)+\frac{f}{2 b}\left(1-\frac{g_{2}}{c_{2}}\right) \ln \left(1+c a+c_{2} a^{2}\right) \\
& -\frac{f}{b}\left(g_{1}-\frac{c}{2}\left(1+\frac{g_{2}}{c_{2}}\right)\right) \frac{2}{\sqrt{4 c_{2}-c^{2}}}\left[\tan ^{-1}\left(\frac{c+2 c_{2} a}{\sqrt{4 c_{2}-c^{2}}}\right)\right. \\
& \left.-\tan ^{-1}\left(\frac{c}{\sqrt{4 c_{2}-c^{2}}}\right)\right] .
\end{aligned}
$$

In the limit $g_{2} \rightarrow 0$ followed by the limit $c_{2} \rightarrow 0$, we find that $E^{(3)}(a) \rightarrow E^{(2)}(a)$.

The mass of the $b$ quark is generally presented in one of two ways. One way is in terms of the " $\overline{M S}$ mass" (which we will denote by $\bar{m}_{b}$ ). In this approach, $\bar{m}_{b}$ is used to fix the boundary condition on the running mass so that

$$
\bar{m}_{b}=m\left(\bar{m}_{b}\right)
$$

and so by eq. (31) we have upon choosing $\mu=\bar{m}_{b}$

$$
M=\frac{\bar{m}_{b}}{E\left(a\left(\bar{m}_{b}\right)\right)} .
$$

The generally accepted value of $\bar{m}_{b}$ is [21]

$$
\bar{m}_{b}=4.18 \mathrm{GeV}
$$

and also for the $S U(3)$ colour gauge group with five flavours using the $\overline{M S} \mathrm{RS}$

$$
\begin{gathered}
b=\frac{23}{6}, \quad c=\frac{29}{23}, \quad c_{2}=1.474789 \\
f=-2, \quad g_{1}=\frac{253}{72}, \quad g_{2}=3.70993 .
\end{gathered}
$$

In refs. [32,33], $c_{2}$ was first calculated while $g_{2}$ appeared first in ref. [34]. The value of the running coupling $a(\mu)$ is generally given at the $Z$ boson mass [21]

$$
\mu=M_{z}=91.1876 \mathrm{GeV}
$$


to be

$$
a\left(\ln \frac{M_{z}}{\Lambda}\right)=\frac{.1185}{\pi}=0.03772
$$

Using the initial conditions above, we demonstrate the scale dependence of the running $\overline{M S}$ coupling $a$ and b-quark mass, $m_{b}$ using eqs. (28,29,30) in Figures 1 and 2 respectively. Based on the 2- and 3-loop results derived above, we also plot the $\mu$ dependence of $M$ of eq. (31) in Figure 3. Here, we find that the 3-loop expression is relatively more scale independent than the 2-loop expression for $M$ as expected, since the exact expression is scale-independent.

To obtain $a\left(\ln \frac{\bar{m}_{b}}{\Lambda}\right)$, needed for $M$ in eq. (61), we use eqs. (29a), (29b) for $X_{0}$ and $X_{1}$ in eq. (28a) with

$$
a\left(\ln \frac{\bar{m}_{b}}{\Lambda}\right) \approx \sum_{n=0}^{1} X_{n}\left(a\left(\ln \frac{M_{z}}{\Lambda}\right) \ln \left(\frac{M_{z}}{\bar{m}_{b}}\right)\right) a^{n}\left(\ln \frac{M_{z}}{\Lambda}\right)
$$

This leads to

$$
a\left(\ln \frac{\bar{m}_{b}}{\Lambda}\right)=.07150
$$

so that in eq. (61) with $E\left(a\left(\ln \frac{\bar{m}_{b}}{\Lambda}\right)\right)$ being approximated by $E^{(2)}\left(a\left(\ln \frac{\bar{m}_{b}}{\Lambda}\right)\right)$ we have

$$
M=15.90 \mathrm{GeV}
$$

In obtaining this value of $M$, we have used $\mu=\bar{m}_{b}$ in eq. (31). In place of this, we could choose $\mu=M_{z}$ with

$$
m\left(\ln \frac{M_{z}}{\Lambda}\right) \approx \bar{m}_{b} \sum_{n=0}^{1} Y_{n}\left(a\left(\ln \frac{\bar{m}_{b}}{\Lambda}\right) \ln \frac{\bar{m}_{b}}{M_{z}}\right) a^{n}\left(\ln \frac{\bar{m}_{b}}{\Lambda}\right)
$$

with $Y_{0}$ and $Y_{1}$ being given in eq. (30). We could also invert eq. (69) so that

$$
m\left(\ln \frac{M_{z}}{\Lambda}\right) \approx \bar{m}_{b}\left[\sum_{n=0}^{1} Y_{n}\left(a\left(\ln \frac{M_{z}}{\Lambda}\right) \ln \frac{M_{z}}{\bar{m}_{b}}\right) a^{n}\left(\ln \frac{M_{z}}{\Lambda}\right)\right]^{-1}
$$

From this equation for $m\left(\ln \frac{M_{z}}{\Lambda}\right)$ and with $E^{(2)}\left(a\left(\ln \frac{M_{z}}{\Lambda}\right)\right)$, we find from eq. (31) that

$$
M \approx 14.70 \mathrm{GeV}
$$

The values of $M$ obtained to second order in perturbation theory in eqs. (68) and (71) using respectively $\mu=\bar{m}_{b}$ and $\mu=M_{z}$ are consistent with the exact expression for $M$ being independent of $\mu$ (see. eq. (32)).

We now can compute the perturbative result for $\Gamma$ given by eq. (23) using $T_{0}, T_{1}$ and $T_{2}$ given in eq. (57). We find that our perturbative result for $\Gamma$ is

$$
\Gamma \approx\left[m\left(\ln \frac{M}{\Lambda}\right)\right]^{5}\left[T_{0}+T_{1} a\left(\ln \frac{M}{\Lambda}\right)+T_{2} a\left(\ln \frac{M}{\Lambda}\right)^{2}\right]
$$


In this equation $m\left(\ln \frac{M}{\Lambda}\right)$ and $a\left(\ln \frac{M}{\Lambda}\right)$ for $M$ given by eqs. (68) and (71), we first use eq. (28) up to $n=1$ (i.e. two-loop order) with $\mu=I M$ and $\mu^{\prime}=\bar{m}_{b}$ and $M_{z}$ respectively. We find that for these two values of $M$, the corresponding values of $\Gamma$ coming from eq. (72) are

$$
\Gamma=615.2 \mathrm{GeV}^{5}
$$

and

$$
\Gamma=648.8 \mathrm{GeV}^{5}
$$

respectively. When $\mu=\bar{m}_{b}$ (as in the case of eq. (73)), we see by eqs. $(6,60)$ that the two loop result for $\Gamma$ corresponds to the RG summed result of eq. (23) but with $M$ replaced by $\bar{m}_{b}$. These two values of $\Gamma$ differ by $2.66 \%$ from the mid point. In Figure 4 , we plot the renormalization scale dependence of the 2-loop perturbative expression as compared to the scale invariant results provided in Eq. (73) and Eq. (74). We unexpectedly find that the Eq. (73) value (where $\mu=\bar{m}_{b}$ in computing $M$ ) intersects with the perturbative curve at $\mu=M$, while the Eq. (74) (where $\mu=M_{z}$ in computing $\left.I M\right)$ value intersects at $\mu=7.6 \mathrm{GeV}$. This key finding shows how our scale invariant expression indicates that a choice of $\mu=M$ renders it equivalent to the two-loop perturbative expression, which implies as to how this renders a choice of $\mu=\bar{m}_{b}$ scale dependent, even though this latter choice sets the RG log terms to zero in the perturbative expression. Overall, our approach eliminates errors in $\Gamma$ due to scale dependence. These errors are typically ascertained using the perturbative expression by variation of the decay rate in the range of $\bar{m}_{b} / 2 \leq \mu \leq 2 \bar{m}_{b}$.

If one were to use three loop contributions to $\beta$ and $\gamma$ but expand $\Gamma$ only to second order in $a$ (since the third order coefficient $T_{3}$ is unknown), then the values of $\Gamma$ corresponding to $\mu=\bar{m}_{b}$ and $\mu=M_{z}$ are

$$
\Gamma=672.4 \mathrm{GeV}^{5}
$$

and

$$
\Gamma=677.8 \mathrm{GeV}^{5}
$$

respectively. These values differ by $0.41 \%$ about the mid point. We thus see that the uncertainty in $\Gamma$ is significantly decreased by going to higher order in perturbation theory.

Even though $\Gamma$ is a physical quantity and is consequently RS independent, any perturbative approximation to $\Gamma$ is $\mathrm{RS}$ dependent. One generally uses the $\overline{M S} \mathrm{RS}$ when doing an explicit calculation as it is the easiest RS to employ, but it should be kept in mind that $c_{i}$ and $g_{i}$ are RS dependent with the dependency of $a, m$ and $T_{n}$ on these parameters fixed by eqs. $(26,34)$ respectively. In refs. [8,9], it is suggested that the optimal values of $c_{i}$ and $g_{i}$ that should be used in any perturbative result is determined by the principle of minimal sensitivity (PMS). This means 
that the optimal values of these parameters that characterize a RS are those whose variation least affects the value of $\Gamma$.

The form of the explicit dependence of $\Gamma$ on $\mu$ appearing in eq. (1) is essential for being able to make use of the RG equation to arrive at the $\mu$-independent result of eq. (23). This form comes from knowing the way in which Feynman diagrams give rise to dependence of $\Gamma$ on $\mu$ at $n$-loop order. Unfortunately, there does not appear to be any way of arriving at a way of seeing in general how $T_{n, k}$ in eq. (1) depends on $c_{i}, g_{i}$ and consequently a summation analogous to that of eq. (7) is not possible. If such a summation were feasible, then the dependence of $\Gamma$ on $c_{i}$ and $g_{i}$ would drop out in a way analogous to the way the dependence of $\Gamma$ on $\mu$ has disappeared in eq. (23).

There are some RS in addition to $\overline{M S}$ that are of particular interest. In the first scheme, the 't Hooft scheme [11], one simply sets $c_{i}=g_{i}=0$. In this case, we see from eqs. $(36,56)$ that $T_{n}=\tau_{n}$ and $S_{n}=\sigma_{n}$. What makes this scheme interesting is that the perturbative series for $\beta$ and $\gamma$ truncates so that

$$
\begin{aligned}
\mu \frac{d a}{d \mu} & =-b a^{2}(1+c a) \\
\mu \frac{d m}{d \mu} & =m f a \Rightarrow \frac{d m}{d a}=-\frac{f}{b} \frac{m}{a(1+c a)}
\end{aligned}
$$

making it possible to find $a(\mu)$ and $m(a(\mu))$. (The Lambert $W$ function occurs in $a(\mu)$ in this RS $[22])$.

In a second scheme, we choose $c_{i}$ and $g_{i}$ so that $T_{n}=0$ in the series for $A_{0}$ for $n \geq 1$ in eq. (7). By eq. (36b), this means that if $T_{1}=0$, then

$$
g_{1}=\frac{-\tau_{1}}{5 \rho \tau_{0}}
$$

while if $T_{2}=0$, then $(36)$

$$
c_{2}+g_{2}=-\frac{2}{5 \rho \tau_{0}}\left[\tau_{2}+\frac{5 \rho g_{1}}{2}\left(-c \tau_{0}+2 \tau_{1}+5 \rho \tau_{0} g_{1}\right)\right]
$$

etc. Having the values of $c_{i}$ and $g_{i}$ fixed in this manner alters the functions $\beta$ and $\gamma$ but reduces $\Gamma$ to the single contribution $T_{0}$ appearing in $A_{0}(a)$.

A related scheme is to choose $c_{i}$ and $g_{i}$ so that the series for $F_{0}$ in eq. (40) terminates at $S_{0}$. By eq. (56) this means that

$$
g_{1}=\frac{-\sigma_{1}}{\rho \sigma_{0}}
$$

for $S_{1}=0$

$$
c_{2}+g_{2}=-\frac{2}{\rho \sigma_{0}}\left(\sigma_{2}+\frac{\rho g_{1}}{2}\left(2 \sigma_{1}-c \sigma_{0}+\rho \sigma_{0} g_{1}\right)\right)
$$

for $S_{2}=0$, etc. Again $\beta$ and $\gamma$ are altered with these conditions, but now the series for $F_{0}$ is reduced to a single term $S_{0}$. 


\section{Discussion}

It is well known that a perturbative evaluation to finite order of radiative corrections to a physical process $\Gamma$ has dependence on the renormalization mass scale $\mu$ and the RS parameters $\left(c_{i}, g_{i}\right)$, all of which are unphysical. However, the way in which one computes Feynman diagrams makes it possible to determine the explicit form of the dependence of $\Gamma$ on $\mu$ at each order of perturbation theory (see. eq. (1)). The RG equation is derived by requiring that this explicit dependence of $\Gamma$ on $\mu$ must be compensated by an implicit dependence of $\Gamma$ on $\mu$ through a "running coupling" $a(\mu)$ and a "running mass" $m(\mu)$. We have shown how this RG equation makes it possible to sum all radiative contributions to $\Gamma$ that depend explicitly on $\mu$ and that upon doing this, the explicit and implicit dependence of $\Gamma$ on $\mu$ cancels so that in place of $\mu, \Gamma$ depends on two mass scales $\Lambda$ and $M$ which are associated with boundary conditions on the running parameters $a(\mu)$ and $m(\mu)$ respectively. In practice, one does not directly deal with $\Lambda$ and $M$, but rather with $a(\mu)$ and $m(\mu)$ at an experimentally determined mass scale. For $a(\mu), \mu$ is generally taken to be $\mathbb{M}_{z}$, while for $m(\mu)$ we use $\mu=\bar{m}_{b}$ which is the " $\overline{M S}$ mass" at which $\bar{m}_{b}=m\left(\bar{m}_{b}\right)$. It is also possible to use $m_{\text {pole }}$, the "pole mass" defined in eq. (28), as the mass scale. This pole mass for the $b$ quark is $m_{\text {pole }}=4.7659 G e V[21]$; we could use $a\left(m_{\text {pole }}\right), m\left(m_{\text {pole }}\right)$ to compute $\Gamma$.

Using these boundary values for $a(\mu)$ and $m(\mu)$, we have been able to deduce $\mathbb{M}$. This involves determining how $a(\mu)$ and $m(\mu)$ vary under changes of $\mu$ using a summation of leading-log, nextto-leading-log etc. contributions to variation of $a(\mu)$; this has been carried out using up to the three-loop contributions to $\beta$ and $\gamma$. This virtually eliminates dependency of $M$ on $\mu$, though there remains $\mathrm{RS}$ dependency residing in $c_{i}$ and $g_{i}$. In addition $\Gamma$ depends on a set of RS independent parameters $\tau_{i}$ and $\sigma_{i}$. One can, in principle, choose $c_{i}$ and $g_{i}$ to reduce $\beta$ and $\gamma$ to a finite series in $a$, or alternatively, these parameters could be chosen to reduce the expansion of $\Gamma$ in eq. (23) to a finite number of terms. It is not clear how the latter choice of RS would affect the nature of the perturbative expansion of $\Gamma$ (in particular how "renormalons" occur [10,13].) It has been proposed $[8,9]$ that the optimal values of $c_{i}$ and $g_{i}$ used to compute $\Gamma$ are those chosen so that $\Gamma$ is stable under small variations away from these values. This is the "principle of minimal sensitivity". This involves satisfying the conditions

$$
\frac{\partial \Gamma}{\partial c_{i}}=0=\frac{\partial \Gamma}{\partial g_{i}}
$$

at each order of perturbation theory. We have not attempted in this paper to select $c_{i}$ and $g_{i}$ using these conditions but rather have just worked in the $\overline{M S} \mathrm{RS}$. In a future study [24], we intend to conduct a systematic error analysis of $\left|V_{u b}\right|$ with considerations of non-perturbative contributions and systematic estimation of scheme dependence errors. We have obtained higher order expressions at 3-loop order and would also draw from estimates $[19,20]$ in order to conduct this analysis.

It would be interesting if there were a way of explicitly summing all of the explicit dependence of $\Gamma$ on $c_{i}$ and $g_{i}$ so as to have a cancellation of the explicit and implicit dependence of $\Gamma$ on these 
parameters, in much the same way that all dependence on $\mu$ has been shown above to cancel. One might expect the final result of such a summation would be the same as what is obtained in the 't Hooft RS in which $c_{i}=g_{i}=0$, so that $T_{n}=\tau_{0}$ and $S_{n}=\sigma_{n}$. Moreover, an extensiom of this type of analysis to the situation in which there is more than one coupling [23] or mass present would be interesting.

\section{Acknowledgements}

We would like to thank A. Kataev for several very helpful suggestions. R. Macleod initiated this work.

\section{References}

[1] S. Weinberg, Phys. Rev. D8, 3497 (1973).

[2] G. 't Hooft, Nucl. Phys. B61, 455 (1973).

[3] M.R. Ahmady, F.A. Chishtie, V. Elias, A.H. Fariborz, N. Fattahi, D.G.C. McKeon, T.N. Sherry and T.G. Steele, Phys. Rev. D66, 014010 (2002) (hep-ph 0203183).

M.R. Ahmady, F.A. Chishtie, V. Elias, A.H. Fariborz, D.G.C. McKeon, T.N. Sherry and T.G. Steele, Nucl. Phys. Proc. Supp. 115, 82 (2003) (hep-ph 0209001).

[4] D.G.C. McKeon, Phys. Rev. D92, 045031 (2015) (hep-th 1503.03823).

[5] F.A. Chishtie, D.G.C. McKeon and T.N. Sherry, Phys. Rev. D94, 054031 (2016) (hep-ph 1512.08713).

[6] F.A. Chishtie and D.G.C. McKeon, Can. J. Phys. 96, 233 (2017) (hep-ph 1610.06487).

[7] A. Czarnecki and K. Melnikov, Phys. Rev. D59, 014036 (1999) (hep-ph 9804215).

[8] P.M. Stevenson, Phys. Rev. D23, 2916 (1981).

[9] D.G.C. McKeon, Can. J. Phys. 61, 564 (1983); 59, 1327 (1981).

[10] G. 't Hooft, The Whys of Subnuclear Physics, Erice 1977, ed. A. Zichichi, Plenum Press N.Y. (1979).

[11] S. Coleman, Aspects of Symmetry, ch. 4, Cambridge University Press, Cambridge 1985.

[12] F.A. Chishtie, V. Elias, R.B. Mann, D.G.C. McKeon and T.G. Steele, Int. J. Mod. Phys. A16, 1681 (2007) (hep-th 0609199). 
[13] M. Beneke, Phys. Rep. 37, 1 (1999) (hep-ph 9807443).

[14] F.A. Chishtie, D.G.C. McKeon and T.N. Sherry, Mod. Phys. Lett. A34, 1950047 (2019) (hep-ph 1806.2534).

[15] W.A. Bardeen, A.J. Buras, D.W. Duke and T. Muta, Phys. Rev. D18, 3998 (1978).

[16] R. Tarrach, Nucl. Phys. B183, 384 (1981).

[17] N.G. Grey, D.J. Broadhurst, W. Grafe and K. Schilcher, Z. Phys. C48, 673 (1990).

D.J. Broadhurst, N. Gray and K. Schilcher, Z. Phys. C52, 111 (1991).

L.V. Avdeev and M.Y Kalmykov, Nucl. Phys. B502, 419 (1997) (hep-ph 9701308).

J. Fleischer, F. Jegerlehner, O.V. Tarasov and O.L. Veretin, Nucl. Phys. B539, 671 (1999) (hep-ph 9803493).

K.G. Chetyrkin and M. Steinhauser, Nucl. Phys. B573, 617 (2000) (hep-ph 9911434).

K. Melnikov and T. van Ritbergen, Phys. Lett. B482, 99 (2000). (hep-ph 9912391).

[18] T. van Ritbergen, Phys. Lett. B454, 353 (1999) (hep-ph 9903226).

T. Seidensticker and M. Steinhauser, Phys. Lett. B467, 271 (1999) (hep-ph 9909436).

[19] T.G. Steele, M.R. Ahmady, F.A. Chishtie and V. Elias, Int. J. Mod. Phys. A15S1B, 642 (2001) (hep-ph 0010045).

[20] M.R. Ahmady, F.A. Chishtie, V. Elias, and T.G. Steele, Phys. Lett. B479 201 (2000) (hep-ph 9910551).

M.R. Ahmady, F.A. Chishtie, V. Elias, A.M. Fariborz, D.G.C. McKeon, T.N. Sherry and T.G. Steele, Phys. Rev. D65 054021 (2002) (hep-ph 0109061).

[21] Particle Data Guide, Phys. Rev. D93, 030001 (2018), Chinese Physics C40, 100001 (2016).

[22] B.A. Magradze, Conf. Prof. C 980598 (1999) hep-th 9808247.

E. Gardi, G. Grunberg and M. Karliner, JHEP 9807:007, (1998) (hep-ph 9806462).

[23] D.G.C. McKeon and Chenguang Zhao, Nucl. Phys. B932, 425 (2018) (hep-ph 1711.04758), Can. J. Phys., 98, 76 (2018) (hep-ph 1807.07523).

[24] F.A. Chishtie, D.G.C. McKeon and T.N. Sherry, in preparation.

[25] P.M. Stevenson, Phys. Rev. D 33, 3130 (1986).

[26] G. 't Hooft in: Deeper Pathways in High Energy Physics, Proc. Obis Scientae (Coral Gables, Florida, 1977). eds. A. Perlmutter and L.F. Scott (Plenum, New York, 1977);

E. de Rafael in Lecture Notes in Physics, Vol. 118, eds. J.J. Alfonso and R. Tarrach (Springer, 
Berlin, 1980).

C. Becchi, S. Narison, E. de Rafael and F.J. Yndurain, Z.Phys. C8, 335, (1981).

D.J. Broadhurst, A.L. Kataev and C.J. Maxwell, Nucl. Phys. B592, 247 (2001) (hep-ph 0007152).

[27] M. Mojaza, S.J. Brodsky and X.G. Wu, Phys. Rev. Lett. 110, 192001 (2013) (hep-ph 1212.0049).

Y. Ma, S. Q. Wang, H. B. Fu, H.H. Ma, S.J. Brodsky and M. Mojaza, Rep. Phys. 78, 126201 (2015) (hep-ph 1405.3196).

[28] A.L. Kataev and S.V. Mikhailov, Phys. Rev. D91, 014007 (2015) (hep-ph 1408.0122).

[29] C.J. Maxwell, Nucl. Phys. B Proc. Suppl. 86, 74 (2000) (hep-ph 9908463).

P. Brooks and C.J. Maxwell, Nucl. Phys. B 780, 76 (2007) (hep-ph 0610137).

[30] M. Akrami and A. Mirjalili, Phys. Rev. D 101, 034007 (2020) (hep-ph 1912.08938).

[31] C.J. Maxwell and A. Mirjalili, Nucl. Phys. B 577209 (2000) (hep-ph 0002204).

[32] O.V. Tarasov, A.A. Vladimirov and A.V. Zharkov, Phys. Lett. 93B, 429 (1980).

[33] S.A. Larin and J. Vermaseren, Phys. Lett. 303B, 334 (1993) (hep-ph 9302208).

[34] O.V. Tarasov, "Anomalous Dimensions of Quark Masses in Three Loop Approximation". Phys. Part. Nucl. Lett 17, 109 (2020) (hep-ph 1910.12231).

[35] P. Marquard, A.V. Smirnov, V.A. Smirnov and M. Steinhauser,Phys. Rev. Lett. 114, 142002 (2015). hep-ph 1502.01030.

P. Marquard, A.V. Smirnov, V.A. Smirnov, M. Steinhauser and D. Wellmann,Phys. Rev. D94, 074025 (2016). hep-ph 1606.06754.

A.L. Kataev and V.S. Molokoedov, Eur. J. Phys. 131, 271 (2016). hep-ph 1511.06898.

JETP Letters 108, 777 (2012) (hep-ph 1811.02867).

Proceedings of Quark 2018 (Valdy, Russia) (hep-ph 1807.05406).

Theor. Math. Phys. 200, 1374 (2019) (hep-ph 1812.09258).

[36] S.G. Gorishnii, A.L. Kataev and S.A. Larin, Phys. Lett. 135B, 457 (1984). 


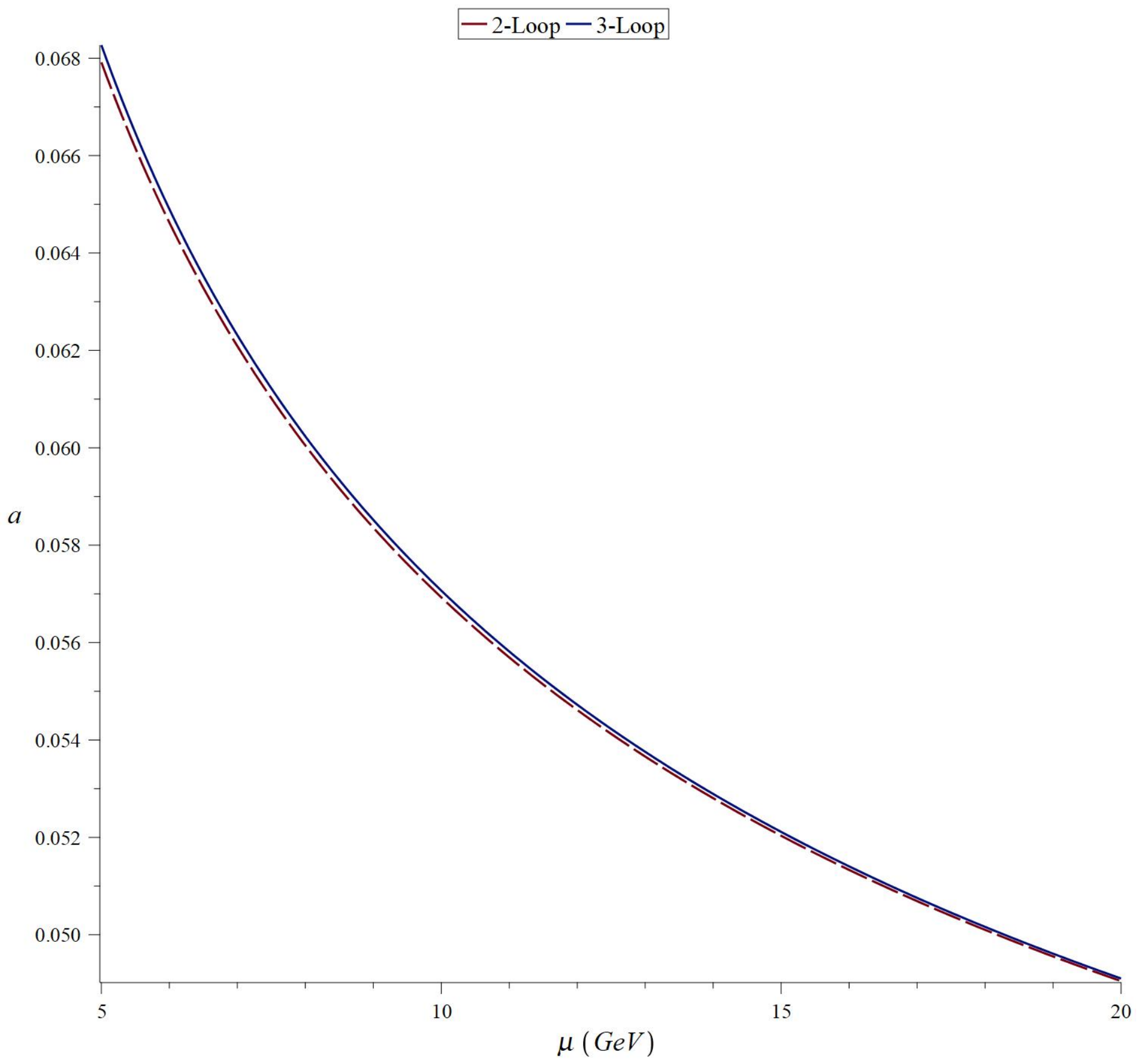

Figure 1: The renormalization scale dependence of strong coupling $a$ in the $\overline{M S}$ scheme at 2 - and 3-loop orders 


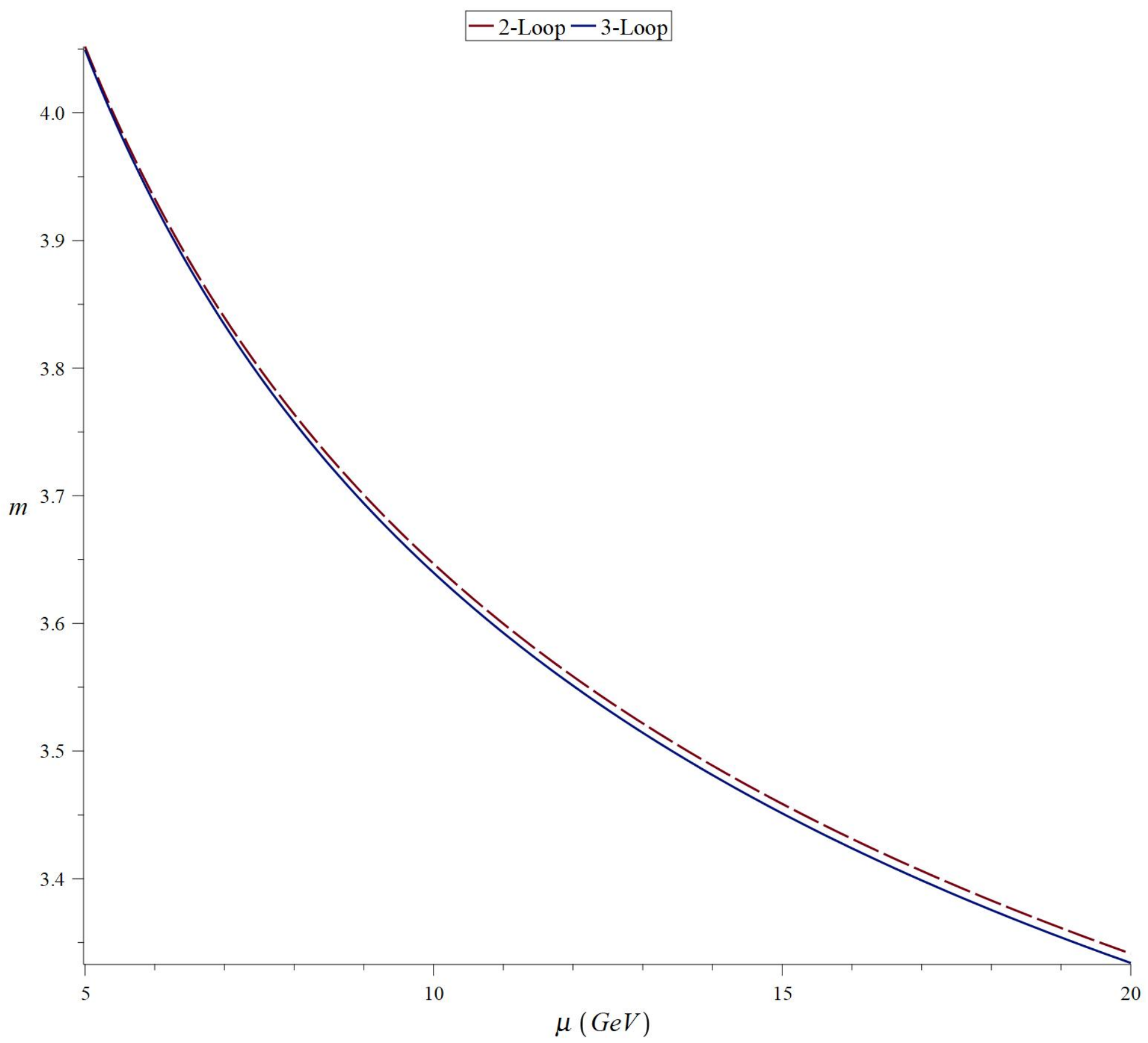

Figure 2: The renormalization scale dependence of $b$-quark mass $m_{b}$ in the $\overline{M S}$ scheme at 2 - and 3-loop orders 


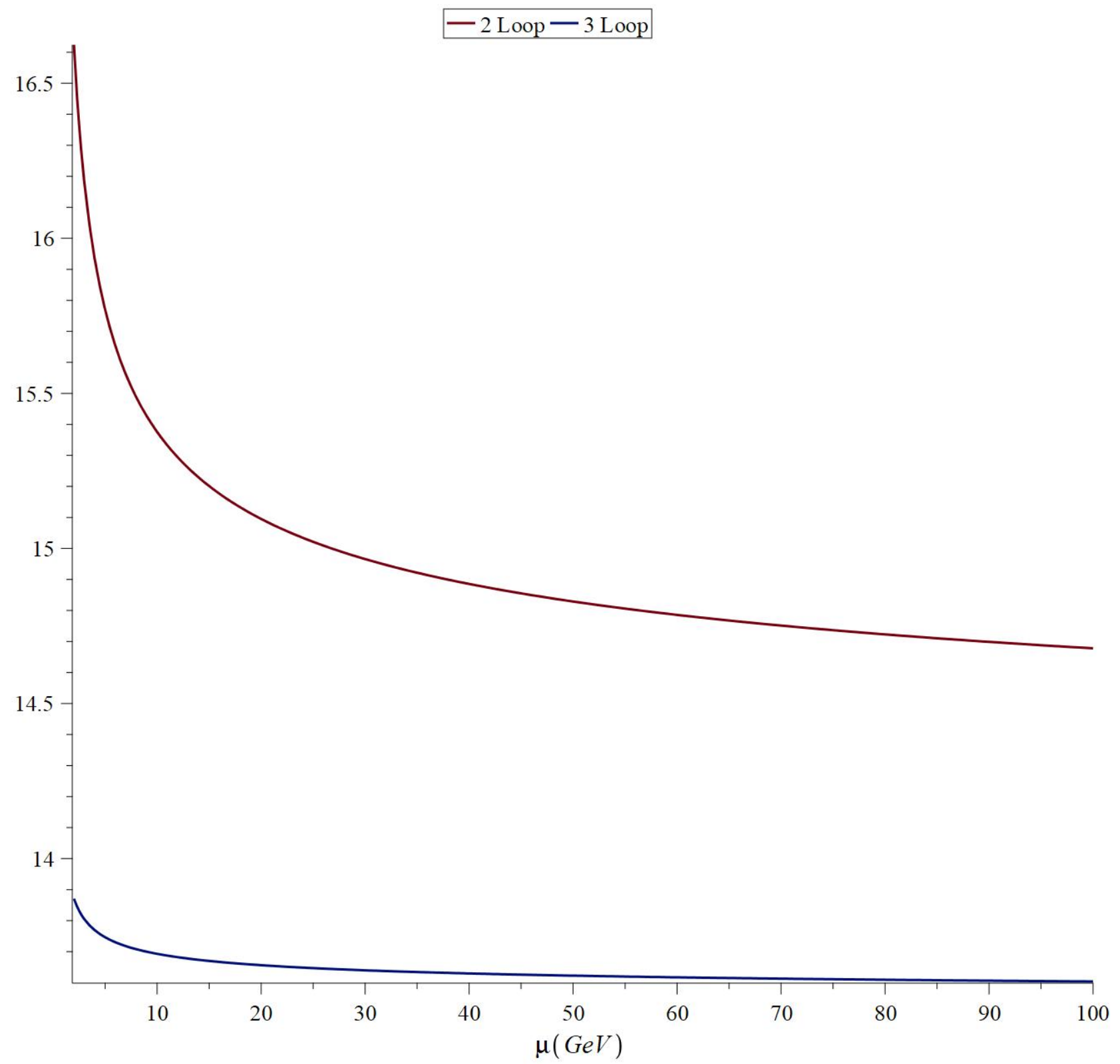

Figure 3: The renormalization scale dependence of $M$ at 2- and 3-loop orders 


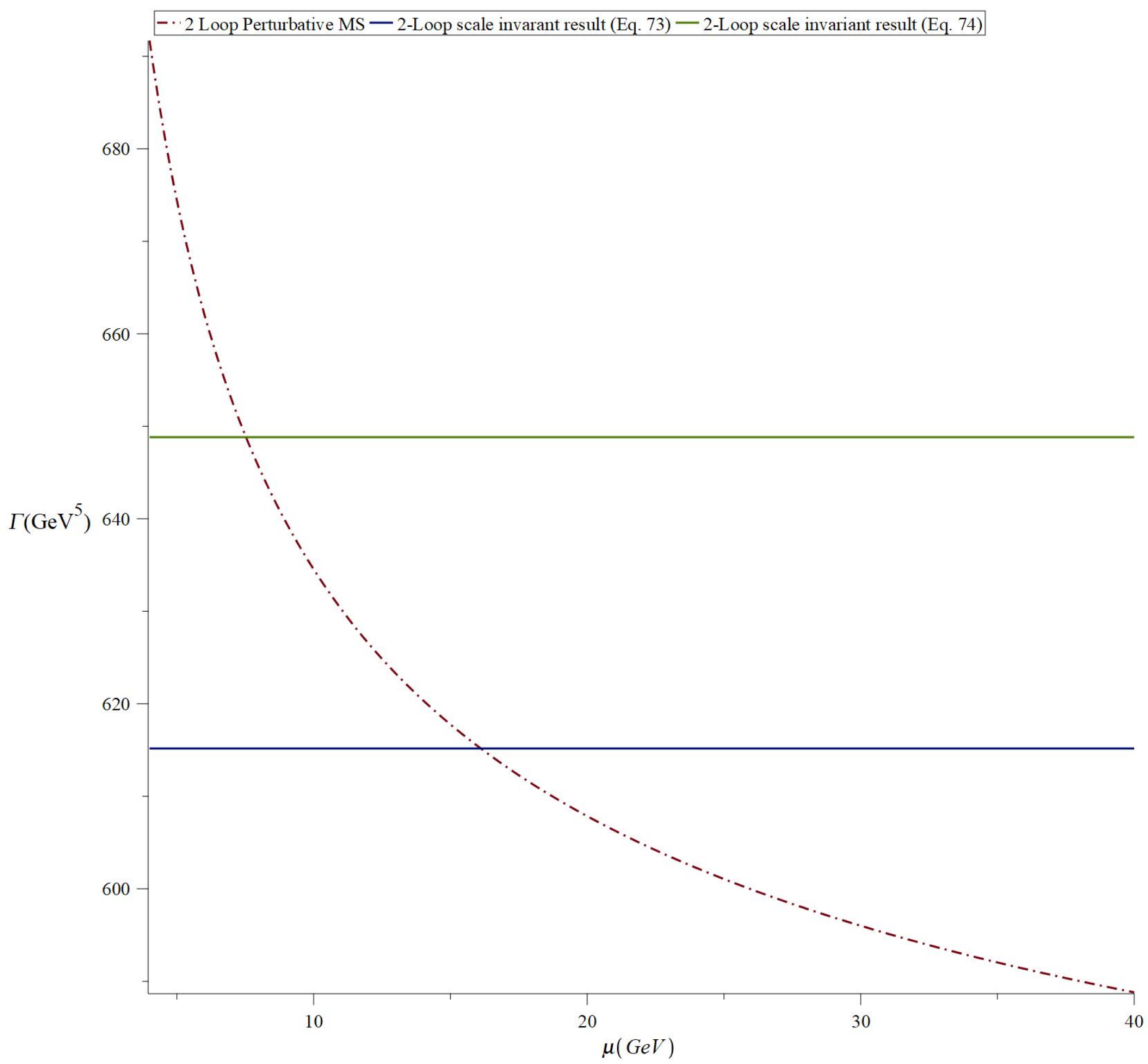

Figure 4: The renormalization scale dependence of 2-loop perturbative $\Gamma$ as compared to the 2-Loop RG scale invariant results from Eqs. 73 and 74. 